Communications in Physics, Vol. 25, No. 3 (2015), pp. 275-282

DOI:10.15625/0868-3166/25/3/6770

\title{
APPEARANCE OF AN ENSEMBLE SOLITARY PULSE TRAIN IN TRANSIENT BACKWARD STIMULATED RAMAN SCATTERING
}

\author{
NGUYEN MANH THANG \\ Academy of Military Science and Technology, Hanoi, Vietnam \\ E-mail: thangnm@jmst.info \\ Received 25 August 2015 \\ Accepted for publication 29 September 2015
}

\begin{abstract}
A self-similarity approximation model for three-wave resonant interaction in transient backward stimulated Raman scattering (BSRS) was derived. Interestingly, as its asymptotic solution, the train of solitary backward Raman pulse train is possibly formed. This result can be considered as an increment of "single" solitons observed previously.
\end{abstract}

Keywords: solito ns, backward Raman scattering, self-similarity.

\section{INTRODUCTION}

Optical solitons is one of the most intriguing subjects in nonlinear optic interactions. Normally, it can be divided into two main branches. The first one results from an exact cancellation between self-phase modulation and group velocity dispersion [1]. This type has been widely studied in both theory and experiment in dispersive media [2-5]. Another one is determined by the balance between energy exchange rates of dispersionless waves of different velocities in threewave resonant interaction $[6,7]$. This structure is ubiquitous in various branches of science as plasma solitons [8-10], optical parametric solitons [11-13], stimulated backward Brillouin scattering [14, 15], solitons in forward stimulated Raman scattering (FSRS) [16-18]. Soliton in backward stimulated Raman scattering (BSRS) was theoretically predicted in 1969 [19] and observed recently in gas filled hollow-core photonic bandgap fibres (HC-PBG) [20]. In this paper, we take advantage of a self-similarity approximation solution analogous to that of transient FSRS to order to consider the transient BSRS interaction problem. The finding solution results in an appearance of the solitary backward Raman pulse train moving superluminously.

\section{THREE-WAVE RESONANT EQUATIONS AND ASYMPTOTIC SOLUTIONS}

It is known that the self-similar solution in transient backward interactions have been theoretically predicted in Brillouin scattering [15,21] and plasma [10]. In this report, we will increment for transient BSRS.

The envelope equations of the three-wave interaction in BSRS are described in Eqs. (1), (2), and (3). In which, the short Stokes pulse $E_{S}$ counter-propagates with respect to a long pump (C)2015 Vietnam Academy of Science and Technology 
pulse $E_{P}$ in resonance with the coherence wave $Q$

$$
\begin{gathered}
\frac{\partial E_{P}}{\partial z}+\frac{1}{c} \frac{\partial E_{P}}{\partial t}=i \kappa_{2}\left(\frac{\omega_{P}}{\omega_{S}}\right) Q E_{S} \\
-\frac{\partial E_{S}}{\partial z}+\frac{1}{c} \frac{\partial E_{S}}{\partial t}=-i \kappa_{2} Q^{*} E_{P} \\
\frac{\partial Q}{\partial t}+\Gamma_{2} Q=-\frac{1}{4} i \kappa_{1}^{*} E_{P} E_{S}^{*}
\end{gathered}
$$

where $\kappa_{1,2}$ are Raman coupling constants and $\Gamma_{2}$ is a dephasing rate of Raman medium depending on pressure and temperature. Here, we assumed that the population inversion of the excited and ground states approximates unity $(n \approx 1)$ while group velocities in a dispersionless medium $v_{P} \approx$ $v_{S} \approx c$. The absorption losses ( $\gamma_{P}$ and $\gamma_{S}$, respectively) and the contribution of Stark-shift $(\Delta)$ are also ignored.

In order to derive an asymptotically self-similar solution for transient BSRS [17], we use the following variables

$$
\begin{gathered}
x=\kappa_{2} z+c \kappa_{2} t ; \tau=c \kappa_{2} t ; \mu=\frac{\Gamma_{2}}{c \kappa_{2}} \\
A_{P}=\sqrt{\frac{\kappa_{1}^{*}}{2 c \kappa_{2}}} E_{P} ; A_{S}=\sqrt{\frac{\kappa_{1}^{*}}{2 c \kappa_{2}}} E_{S} ; Q=i \rho,
\end{gathered}
$$

where the variables $x$ and $\tau$ are linked to Stokes pulses. The longitudinal coordinate- $\tau$ describes the total distance of Stokes pulse passed an active Raman medium. The coordinate-x describes the distance between its front and maximum while $\mu$ is a normalized dephasing rate (Raman linewidth). The imaginary coherence means that the resonance is complete in three-wave interaction. In this context, we choose $E_{P, S}$ real. It is also noted that $E_{P, S}$ could be real without lack of generality in Ref. [10,22]. Eqs. (1), (2), and (3) can be rewritten as below

$$
\begin{gathered}
2 \frac{\partial A_{P}}{\partial x}+\frac{\partial A_{P}}{\partial \tau}=\rho A_{S}, \\
\frac{\partial A_{S}}{\partial \tau}=-\rho A_{P} \\
\frac{\partial \rho}{\partial x}+\left(\frac{\partial \rho}{\partial \tau}+\mu \rho\right)=-\frac{1}{2} A_{S} A_{P}
\end{gathered}
$$

The backward amplification process can be relatively divided into two regimes: linear and nonlinear regimes $[10,21]$. The linear stage occurs at low pump energy levels or short interaction time, which results in near uniformly amplified seed Stokes and its position is constant in time or moves with the light speed in vacuum.

The nonlinear stage takes place at higher pump energy levels or longer interaction time. Signal (seed Stokes) pulses become resharpening, narrowing, and moving with a superluminal speed. Hence, Stokes pulse's maximum approaches its front or the distance-x will be gradually small compared with the total distance- $\tau$. With the characteristics of the backward amplification, 
a consequence is given: the dependence of all amplitudes on the coordinate- $\tau$ can be negligible in comparison to the coordinate- $x[10,22,23]$ :

$$
\frac{\partial\left(A_{P, S}, \rho\right)}{\partial \tau}<<\frac{\partial\left(A_{P, S}, \rho\right)}{\partial x}
$$

On the other hand, due to a very high Raman gain, HC-PBG can support a transient SRS regime in an ultra-long pump pulse duration (several ns) which is several orders longer than previous study $[18,24]$. Therefore, we can use an approximation $\mu \approx 0$. From Eq. (7) and above analysis, Eqs. (4), (5), and (6) can be reformulated as

$$
\begin{gathered}
\frac{\partial A_{P}}{\partial x}=\frac{1}{2} \rho A_{S} \\
\frac{\partial A_{S}}{\partial \tau}=-\rho A_{P} \\
\frac{\partial \rho}{\partial x}=-\frac{1}{2} A_{S} A_{P}
\end{gathered}
$$

The conservation of photon and optical phonon numbers gives us

$$
\frac{A_{P}^{2}(x, \tau)}{\omega_{P}}+\frac{\rho^{2}(x, \tau)}{\Omega}=\frac{a_{0}^{2}(0, \tau)}{\omega_{P}},
$$

where $\omega_{P}-\omega_{S}=\Omega$ (frequency of coherence wave) and $a_{0}(0, \tau)$ is the temporal amplitude of pump field at the input position $\mathrm{x}=0$. We can rewrite Eq. (11) in term of a new variable $\psi(x, \tau)$ as follows

$$
\begin{aligned}
A_{P} & =a_{0} \cos (\psi / 2), \\
\rho & =\sqrt{\frac{\Omega}{\omega_{P}}} a_{0} \sin (\psi / 2)
\end{aligned}
$$

Substituting Eq. (12) into Eq. (11) we obtain the Stokes amplitude $A_{S}$

$$
A_{S}=-\sqrt{\frac{\omega_{P}}{\Omega}} \frac{\partial \psi}{\partial x}
$$

We can also replace Eq. (12) and Eq. (13) into Eq. (9)

$$
\frac{\partial^{2} \psi}{\partial x \partial T}=\sin \psi \text { with } T=\frac{\Omega}{2 \omega_{P}} \int_{-\infty}^{\tau} a_{0}^{2}\left(0, \tau^{\prime}\right) d \tau^{\prime}
$$

Equation (14) is the well-known sine-Gordon equation. It supports the approximated solution by a self-similar function $\mathfrak{I}(Z)=\psi(x, T)$, where $Z=2 \sqrt{x T}$ satisfies the ordinary differential equation

$$
\frac{d^{2} \mathfrak{I}}{d Z^{2}}+\frac{1}{Z} \frac{d \mathfrak{I}}{d Z}=\sin \mathfrak{I}
$$

The solution of Eq. (15) depends on the boundary conditions. In the context of our consideration, it is given by $\psi(0+)=0$, meaning that there is no coherence before the interaction between pump wave and seed Stokes. $\psi^{\prime}(0)=\varepsilon$ ( $\varepsilon$ is very small constant) defines the initial amplitude of seed Stokes. The important characteristic of the self-similar function $\mathfrak{I}(Z)$ is its amplitude, which is oscillates asymptotically to the equilibrium value- $\pi$, so called " $\pi$-pulse solution". 


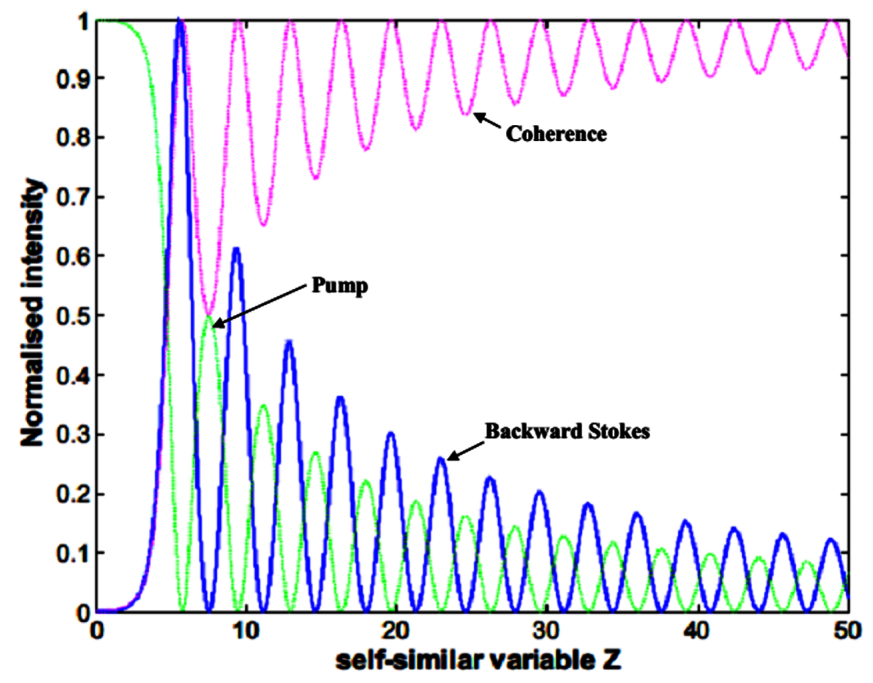

Fig. 1. Normalized photon densities: pump density- $f_{P}$, backward Stokes density- $f_{S}$, and coherence density- $f_{C}$ are shown as above

In the Fig. 1, the parameters are denoted as below:

$$
f_{P}=\frac{A_{P}^{2}}{a_{0}^{2}}=\cos ^{2}(\psi / 2) ; \quad f_{C}=\frac{A_{S}^{2}}{\left(A_{S}^{2}\right)_{\max }} ; \quad f_{S}=\frac{\Omega}{\omega_{P}} \frac{\rho^{2}}{a_{0}^{2}}=\sin ^{2}(\psi / 2) .
$$

The dynamical evolution of pump, backward Stokes, and coherence in respect to the selfsimilar variable $Z$ are described by normalized energy densities in Fig. 1 . It shows that in the transient BSRS, it is possible to appear a series of oscillations caused by the phase shift- $\pi$ from self-similar function $\mathfrak{I}(Z)$. The physical origin of this behavior can be understood as an ability of retaining "memory" or coherence after passing of applied fields in the transient BSRS.

\section{GENERATION OF SOLITARY PULSE TRAIN IN REAL CONFIGURATION}

HC-PBG as a microcell offers an excellent guiding structure: i) the diffraction-free long interaction length allows light to be tightly confined during its propagation and ii) the flexibility in designing the position of the guidance band allows us to remove unwanted higher order Raman components [24]. These excellent features make it to be an excellent candidate for the investigation of light - gas interaction inside its hollow-core. The interaction between pump wave $E_{P}$ (green curve) and backward Stokes wave (red curve) $E_{S}$ in $\mathrm{H}_{2}$ gas filled HC-PBG is numerically calculated in Fig. 2. For simplicity, we assumed that both pump and seed shapes to be Gaussian with a pump width of $35 \mathrm{~ns}$ (few times as long as seed pulse $8 \mathrm{~ns}$ ). It is noted that the multi-peak behavior is independent to the temporal shape of seed Stokes [25]. In our study, the HC-PBG consists of a core with the core's radius and length of $5 \mu \mathrm{m}$ and $1.4 \mathrm{~m}$, respectively. The propagation constants of Stokes and pump waves $\beta_{S}=5.5 \times 10^{6} \mathrm{~m}^{-1} ; \beta_{P}=5.9 \times 10^{6} \mathrm{~m}^{-1}$ [20], respectively. The seed energy is assumed to be $0.1 \mu \mathrm{J}$ and the gas pressure is 5 bar. The normalized dephasing rate $\mu$ is chosen as 0.0145 while the rotational Raman strength $\kappa_{1}^{*}=7.4 \times 10^{-8}\left(\mathrm{~m}^{2} / \mathrm{V}^{2}\right)$. The 
orthogonal- $\mathrm{H}_{2}$ gas density is chosen as $62 \%$ of the total gas density at the room temperature. The pump energy is assumed to be $18 \mu \mathrm{J}$, where either it supports a transient regime [26] or the pump intensity is strong enough to create a series of oscillations.

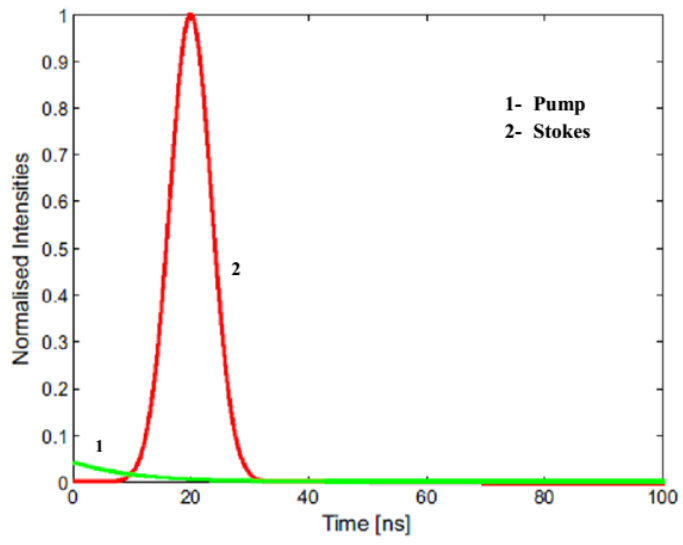

a) $\mathbf{t}=\mathbf{0 n s}$

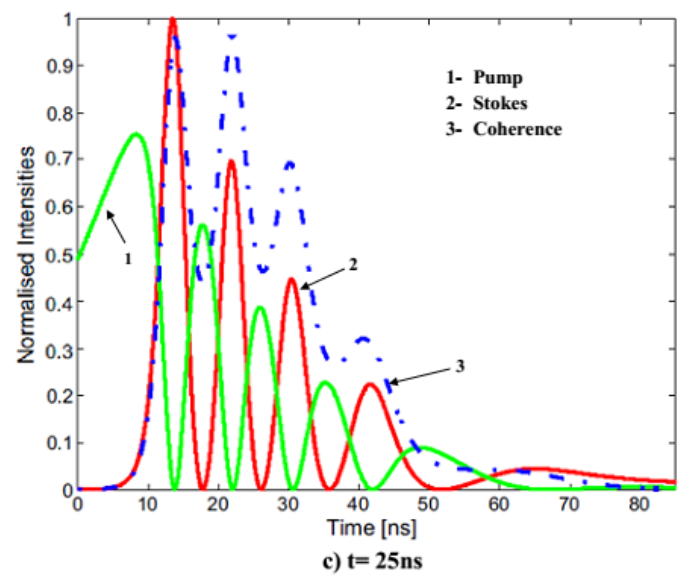

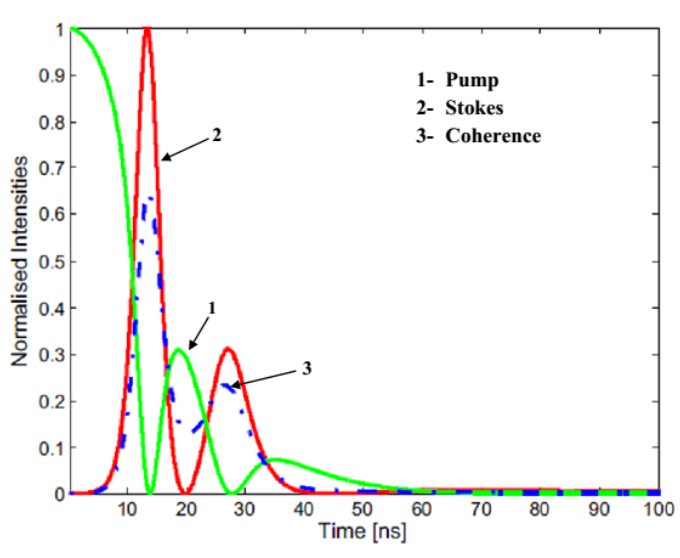

b) $t=10 \mathrm{~ns}$

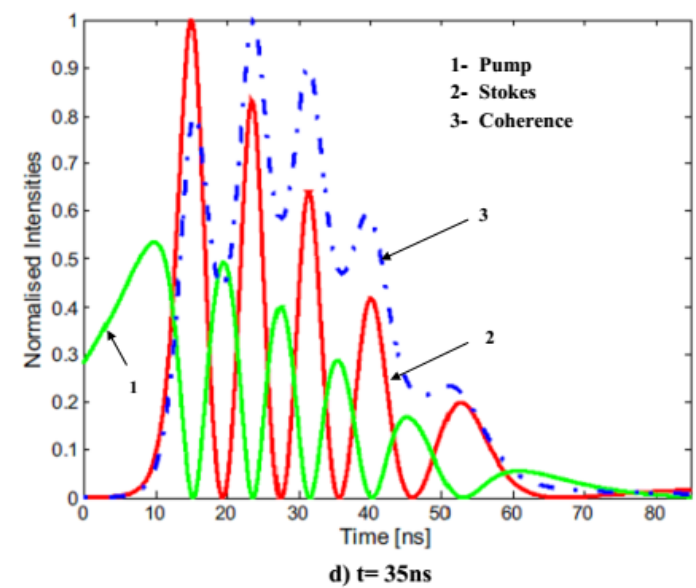

Fig. 2. Normalized evolution of the transient BSRS at different interaction times $t=0 \mathrm{~ns}$; $t=10 \mathrm{~ns} ; t=25 \mathrm{~ns} ; t=35 \mathrm{~ns}$. In which 1-Pump pulse, 2-Stokes and 3-Coherence

The temporal evolution of the three-wave resonant interaction process is described as following: At initial time $t=0$, no interaction between pump and seed Stokes occurs, leading to have no coherence. When $t>0$, seed pulse is amplified by counter-propagating pump wave, coherence appears with a time-delay with seed pulse. When interaction time increases, seed pulse is amplifying. As pump intensity is not strong enough to pass the effect of dephasing behavior (or relaxation), Stokes pulse will be close uniformly amplified like as experiment in linear stage. When pump intensity is sufficient strong, it can pass the effect of dephasing [27]. It also means that the transient SRS regime is broadened for pump width. The multi-peak appearance can be understood by considering the energy flow among the pump, Stokes and coherence. As Stokes and coherence fields grow, the pump field tends to be depleted toward zero and forming an energy flow 
back into the pump field, its phase is possible to be shifted- $\pi$. The growth of pump field causes the depletion of Stokes and coherence fields at Stokes' trailing edge. Results in Stokes pulse is sharpened at its front and reduced quickly at its trailing or Stokes pulse become narrowing. When pump duration (interaction time) is equal or shorter than Stokes' width, Stokes pulse will follow a single pulse process like as Ref. [20] and doesn't have any other peaks. When pump duration is several times larger than Stokes' width, it is possible to emerge another peak after the depletion of the first peak caused by an ability of remaining "coherence memory" after the applied fields passed. This second peak is also amplified gradually the same as the first one and so on. Consequently, as the interaction time is long enough, we can observe multi-oscillation behavior. Of course, peak number will be increased with the increasing of pump duration and intensity. Temporal evolution is gradually represented in Fig. 2 (a-d). At interaction time $t=35 \mathrm{~ns}$ (about pump width), Stokes structure theoretically has four additional peaks (Fig. 2d) with pulse durations about $4 \mathrm{~ns}, 4.5 \mathrm{~ns}$, $5 \mathrm{~ns}, 5.5 \mathrm{~ns}$ corresponding to the first, second, third and fourth order ones.

Fig. 3 shows the backward amplification by the increasing of pump energy. When pump energy increases, all of peaks become resharpening, narrowing, superluminous moving. At the pump energy $14 \mu \mathrm{J}$, the pulse durations are about $4.3 \mathrm{~ns}, 5 \mathrm{~ns}, 5.6 \mathrm{~ns}, 6.3 \mathrm{~ns}$ corresponding to the first, second, third and fourth seed's order ones. Moreover, these peaks move slowly gradually with the increasing of pump energy, earlier peaks give slower superluminal speeds. When pump energy is increased to a relatively high value, peaks will stop resharpening, moving superluminally and reach an asymptotically symmetric shape (hyperbolic-secant).

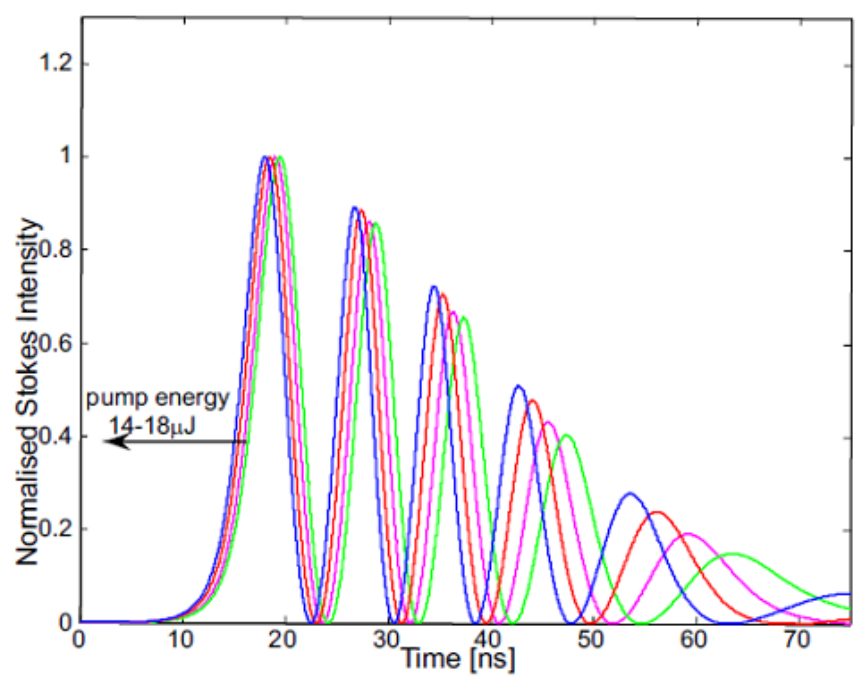

Fig. 3. Resharpening, narrowing via pump energy increasing: black arrow shows the direction of pump energy in the range $14 \mu \mathrm{J}-18 \mu \mathrm{J}$

In the limit of high pump energy or long interaction time, Eq. (15) gives the $2 \pi$-pulse solution, $\mathfrak{I}=4 \arctan (\exp (Z))$. Substituting it into Eqs. (12), (13) we have:

$$
A_{P}=a_{0} \tanh (Z)
$$




$$
\begin{aligned}
\rho & =\sqrt{\frac{\Omega}{\omega_{P}}} a_{0} \operatorname{sech}(Z) \\
A_{S} & =-2 \sqrt{\frac{\omega_{P}}{\Omega}} \operatorname{sech}(Z)
\end{aligned}
$$

Equation (18) indicates an asymptotic state from the evolution of Stokes envelope. For simplicity, assuming the pump wave has a very long duration with constant intensity (as a cw beam). The temporal shape of backward seed Stokes is arbitrary but significantly shorter than that of pump.

The behavior of pulse train in the limit of high pump energy or very long interaction length (an asymptotic state) is shown in Fig. 3. In this regime, the speed of Stokes and coherence are equal. Stokes structure is resharpened asymptotically to a hyperbolic-secant peak train like as a further development of single solitary problem [20].

\section{CONCLUSIONS}

We developed the self-similarity approximation solution for transient backward stimulated Raman scattering equations. The multi-peak appearance and dynamics in the highly coherent regime is analyzed in detail. In the limit of high pump energy or long interaction time, the train of solitary pulses moving with superluminous velocities can be generated. The gas filled HC-PBG configuration is also proposed for observing this new phenomenon. Of course, it is also applied for other gases having a high Raman gain.

\section{ACKNOWLEDGMENTS}

The author wish to thank to Prof. Philip Russell, Dr. A. Walser, and Dr. Amir Abdolvand for useful discussions. This work is partially supported by Max-Planck Research Grants.

\section{REFERENCES}

[1] G. P. Agrawal, Nonlinear Fiber Optics, Academic press, 2007.

[2] A. Hasegawa and F. Tappert, Applied Physics Letters 23 (3) (1973) 142-144.

[3] L. F. Mollenauer, R. H. Stolen, and J. P. Gordon, Phys. Rev. Lett. 45 (13) (1980) 1095.

[4] V. N. Serkin and A. Hasegawa, Phys. Rev. Lett. 85 (21) (2000) 4502.

[5] P. Grelu and N. Akhmediev, Nature Photonics 6 (2) (2012) 84-92.

[6] J. Armstrong, S. S. Jha, and N. Shiren, Quantum Electronics, IEEE Journal of 6 (2) (1970) 123-129.

[7] D. J. Kaup, A. Reiman, and A. Bers, Reviews of Modern Physics 51 (2) (1979) 275-309.

[8] K. Nozaki and T. Taniuti, Journal of the Physical Society of Japan 34 (3) (1973) 796-800.

[9] H. J. LEE, J. KIM, and H. SUK, Journal of the Korean Physical Society 44 (5) (2004) 1246-1249.

[10] V. Malkin, G. Shvets, and N. Fisch, Phys. Rev. Lett. 82 (22) (1999) 4448.

[11] S. Trillo, Optics Letters 21 (15) (1996) 1111-1113.

[12] A. Picozzi and M. Haelterman, Optics letters 23 (23) (1998) 1808-1810.

[13] A. Degasperis, M. Conforti, F. Baronio, and S. Wabnitz, Phys. Rev. Lett. 97 (9) (2006) 093901.

[14] F. Baronio, M. Andreana, M. Conforti, G. Manili, V. Couderc, C. De Angelis, and A. Barthélémy, Optics express 19 (14) (2011) 13192-13200.

[15] E. Picholle, C. Montes, C. Leycuras, O. Legrand, and J. Botineau, Physical review letters 66 (11) (1991) 1454.

[16] A. Luimes and K. Drühl, Optics communications 116 (1) (1995) 279-290.

[17] C. R. Menyuk, D. Levi, and P. Winternitz, Phys. Rev. Lett. 69 (21) (1992) 3048.

[18] A. Nazarkin, A. Abdolvand, A. Chugreev, and P. S. J. Russell, Phys. Rev. Lett. 105 (17) (2010) 173902.

[19] M. Maier, W. Kaiser, and J. Giordmaine, Phys. Rev. 177 (2) (1969) 580. 
[20] A. Abdolvand, A. Nazarkin, A. Chugreev, C. Kaminski, and P. S. J. Russell, Phys. Rev. Lett. 103 (18) (2009) 183902.

[21] D. Bobroff and H. Haus, Journal of Applied Physics 38 (1) (1967) 390-403.

[22] J. Coste and C. Montes, Phys. Rev. A 34 (5) (1986) 3940.

[23] V. A. Gorbunov, Soviet Journal of Quantum Electronics 14 (8) (1984) 1066.

[24] F. Couny, O. Carraz, and F. Benabid, JOSA B 26 (6) (2009) 1209-1215.

[25] R. L. Carman, F. Shimizu, C. Wang, and N. Bloembergen, Phys. Rev. A 2 (1) (1970) 60.

[26] M. Raymer and J. Mostowski, Phys. Rev. A 24 (4) (1981) 1980.

[27] V. Fuchs and G. Beaudry, Journal of Mathematical Physics 16 (3) (1975) 616-619. 